\title{
FCGR2C Gene
}

National Cancer Institute

\section{Source}

National Cancer Institute. FCGR2C Gene. NCI Thesaurus. Code C38556.

This gene is involved in the initiation of host immune responses ag ainst pathogens and phagocytosis. 